\title{
Selección denso-dependiente de microhabitat en Galaxias platei: un estudio experimental
}

\section{Density-dependent microhabitat selection in Galaxias platei: an experimental study}

\author{
Catterina Sobenes ${ }^{1,2 *}$, Oscar Link ${ }^{3}$ \& Evelyn Habit ${ }^{1,4}$ \\ ${ }^{1}$ Centro de Ciencias Ambientales EULA-Chile, Universidad de Concepción, Barrio Universitario s/n, casilla 160-C, Concepción, \\ Chile. \\ 2Departamento de Ingeniería Ambiental y Recursos Naturales, Facultad de Ingeniería, Universidad Católica de la Santísima \\ Concepción, Alonso de Rivera 2850, Concepción, Chile. \\ ${ }^{3}$ Departamento de Ingeniería Civil, Universidad de Concepción, Barrio Universitario s/n, casilla 160-C, Concepción, Chile. \\ ${ }^{4}$ Centro de Investigaciones en Ecosistemas Patagónicos (CIEP), Coyhaique, Chile. \\ *E-mail: csobenes@udec.cl.
}

\begin{abstract}
RESUMEN
Galaxias platei es la especie nativa de mayor distribución en la Patagonia Chilena, habitando ambientes fluviales y principalmente lacustres. Utiliza variados hábitats, desde plataformas de arcilla consolidada a ambientes de sustrato fino y otros con presencia de macrófitas, vegetación leñosa o sustrato rocoso grueso. En este trabajo analizamos experimentalmente la selección de microhábitat de G. platei y su dependencia con la disponibilidad de refugio y la densidad de peces que lo utilizan. Para ello diseñamos experimentos con distintos niveles de refugio a diferentes densidades. Se recrearon microhábitats con refugio de canto rodado (sustrato grueso) y refugio de vegetación acuática, y microhábitats sin refugio de grava (sustrato fino) y piedra plana simulando sustrato de arcilla consolidada. Las densidades probadas fueron 4, 8, 12 y 16 individuos. Encontramos que en todas las densidades, G. platei selecciona microhábitats con refugio y que al aumentar la densidad se produce un cambio en la preferencia desde microhábitats con vegetación hacia sustratos de canto rodado. Por el contrario, en todas las densidades probadas, los microhábitats sin refugio fueron rechazados significativamente. Tanto la selección denso-dependiente de microhábitats, como la tendencia a un comportamiento gregario a mayor densidad de individuos, sugieren un comportamiento antidepredatorio de la especie.
\end{abstract}

Palabras Claves: Experimentación, refugio, interacción intraespecífica, peces nativos, Patagonia.

\begin{abstract}
Galaxias platei is the most broadly distributed fish specie in the Chilean Patagonia that inhabits in rivers and mainly in lakes systems. It occurs on varied habitats from clay platforms to thin substrates with presence of macrophytes, large woody debris and big boulders substrata. In this work we experimentally analyzed the microhabitat use of G. platei with dependence of refuge availability and fish density. We conduct experiments at different refuge levels for different fish densities. We build refuge microhabitat using boulders (thick substrate) and aquatic vegetation, and microhabitat without refuge using gravel (thin substrate) and slab stone to simulate a clay substrate. Fish densities tested were 4, 8, 12 and 16 individuals. $G$. platei select microhabitats with refuge at all densities tested, changing preference from vegetation to boulders at increasing density. Whereas, significant selection against to all microhabitats without refuge at all densities. Density dependent use of microhabitat and the tendency to a gregarious behavior at high fish density, suggest an antidepredatory behavior of the specie.
\end{abstract}

KEYWORDS: Experimentation, refuge, intraspecific interaction, native fishes, Patagonia.

\section{INTRODUCCIÓN}

La especie nativa Galaxias platei Steindachner 1898 es un pez de amplia distribución en aguas continentales del sur de Sudamérica (McDowall 2006). Dada su gran representatividad en variados ambientes de la Patagonia Chilena en los últimos años se ha puesto particular atención sobre ella, estudiando sus patrones de distribución y abundancia a nivel genético y poblacional (Ruzzante et al. 2008; Zemlack et al. 2008, 2011; Jara-Seguel et al. 
2008; Habit et al. 2010, 2012; Correa \& Hendry 2012). Sin embargo, existe poco conocimiento sobre su historia de vida (García de Leaniz et al. 2010; Penaluna et al. 2009), lo que constituye uno de los principales obstáculos para su conservación y para la formulación de estrategias de conservación efectivas (Habit et al. 2010; McIntosh et al. 2010; García de Leaniz et al. 2010; García et al. 2012a). En Chile, G. platei se distribuye desde la cuenca del río Valdivia (39 $9^{\circ}$ Lat. S) por el Norte hasta Tierra del Fuego (54 Lat. S.) (Cussac et al. 2004). Es una especie frecuente en ríos y lagos postglaciales de la Patagonia (Ruzzante et al. 2008; Cussac et al. 2009; Zemlak et al. 2008, 2011; Habit et al. 2010), cuya distribución se ha visto fuertemente afectada por la invasión de salmónidos (Habit et al. 2010, 2012; Correa \& Hendry 2012). En algunos sistemas su abundancia es inversamente proporcional a la abundancia de salmónidos (Habit et al. 2010), sugiriendo una competencia por hábitats, lo que podría resultar en el desplazamiento de la especie nativa de sus hábitats óptimos.

En términos teóricos los peces utilizan hábitats que les son energéticamente óptimos, es decir, donde obtienen la máxima cantidad de energía posible para crecer y reproducirse, evitando ser depredados (MacArthur \& Pianka 1966; Charnov 1976; Werner et al. 1983). Tales hábitats serían los preferidos y por ende seleccionados entre los existentes en el ambiente (Fretwell \& Lucas 1970). En ese contexto, los peces utilizarían hábitats menos óptimos sólo en condiciones de falta o degradación de la calidad de sus hábitats preferentes. Una de las razones por las que un hábitat óptimo puede disminuir de calidad es el incremento en la densidad de individuos en el mismo. En tal caso, los peces tenderían a desplazarse entre hábitats, buscando mantener su bienestar (Fretwell \& Lucas 1970). A este comportamiento se lo define como selección de hábitat denso - dependiente (Rangeley \& Kramer 1998). Así, la densidad de los peces en conjunto con la disponibilidad de hábitats con refugio y alimento, definen las tácticas anti depredatorias de la especie (Pitcher 1983; Griffiths \& Armstrong 2002; Davey et al. 2009).

La selección de hábitat, mediada por factores de densidad poblacional y disponibilidad de hábitats, ha sido evaluada preferentemente en peces de interés comercial ya que ello ha permitido definir las densidades para distintos sistemas acuáticos (Rangeley \& Kramer 1998; Armstrong \& Griffiths 2001; Griffiths \& Armstrong 2002; Davey et al. 2005, 2009; Uglem et al. 2009; Camp et al. 2011). En otros casos se han realizado estudios de selección de hábitats denso-dependiente orientados a comprender las relaciones interespecíficas entre especies invasoras y especies nativas, como es el caso de salmónidos y galáxidos en Nueva Zelanda (McIntosh et al. 1994; Townsend 2003; Bonnett \& McIntosh 2004; McDowall 2006; Crow et al. 2010). Para especies de peces nativos chilenos, los estudios de uso de hábitats son escasos (Barriga et al. 2002; Penaluna et al. 2009; García et al. 2012a, 2012b), y además, ninguno de ellos ha incluido la selección denso-dependiente como variable de interés.

El uso de hábitat de G. platei se ha descrito en ríos y en lagos. Galaxias platei utiliza variados hábitats, desde plataformas de arcilla consolidada a ambientes de sustrato fino y otros con presencia de macrófitas, vegetación leñosa o sustrato rocoso grueso. En ríos se le encuentra ocupando zonas de rápidos y pozas (García et al. 2012a). En lagos se le encuentra en distintos sistemas, desde oligotróficos a mesotróficos, y de alta turbidez de origen glaciar (Habit et al., 2010, 2012), los que utilizaría como refugio ante depredadores (Milano et al. 2002). Está adaptada tanto morfológica como fisiológicamente a la vida bentónica, de baja temperatura y luz, características típicas de los lagos de origen glaciar de la Patagonia (Milano et al. 2002, 2006; Cussac et al. 2004). Los juveniles habitan en distintos tipos de sustrato, pero siempre usando preferentemente microhábitats con refugios como troncos sumergidos o abundante materia orgánica como hojas proveniente de la vegetación ripariana (Habit et al. 2012). Los adultos de mayor talla $(400 \mathrm{~mm})$ se han encontrado en la zona eufótica de los lagos postglaciales (Milano et al. 2002) y bajo la termoclina a profundidades de hasta $80 \mathrm{~m}$ (Barriga et al. 2002; Habit et al. 2012). De esta manera, el factor que determina un microhábitat óptimo para esta especie, principalmente en su estado de juvenil, parece ser el refugio, el cual será suficiente y de buena calidad dependiendo de la densidad de individuos que lo utilizan.

En el presente estudio postulamos que el uso de microhábitat de la especie nativa G. platei depende de la disponibilidad de hábitats con refugio y de la densidad de peces que lo utilizan. Para ello diseñamos experimentos para poner a prueba el uso de microhábitats con distintos niveles de refugio a diferentes densidades de G. platei.

\section{MATERIALES Y MÉTODOS}

\section{Colecta de GalaXias PLATEI}

Colectamos individuos de G. platei de longitud total entre $70-140 \mathrm{~mm}$, con pesca eléctrica (Smith-Root), en el río San Pedro de la cuenca del río Valdivia durante el mes de Septiembre del año 2010 (Pesca de Investigación Res. Ex. $\mathrm{N}^{\circ} 55 / 2010$ ). Los peces fueron trasladados en tanques de 1201 con aireación hasta el Laboratorio de Ingeniería Hidráulica y Ambiental de la Universidad de Concepción, y dispuestos en tres acuarios de 701 (R.N.A N ${ }^{\circ}$ 21750/2011). La calidad del agua se mantuvo mediante filtros biológicos sumergidos y sustitución de agua de un 10\% del volumen 
total a la semana. Los peces fueron aclimatados durante 1 mes y alimentados con alimento granulado $(1 \mathrm{~mm})$ para peces de acuario comercial (Sobenes et al, 2012).

\section{ACUARIO EXPERIMENTAL}

Los experimentos fueron realizados en un acuario de vidrio de $1 \times 1,5 \times 0,6 \mathrm{~m}$, con agua a temperatura ambiente entre 14 $-16^{\circ} \mathrm{C}$ y un fotoperiodo con luz fluorescente (12 h luz, 12 $\mathrm{h}$ oscuridad) conectado a un timer eléctrico. Se ubicó en una esquina del laboratorio con un panel lateral adicional, para evitar cualquier interferencia externa sobre la conducta de los peces, quedando un lado del acuario para observación. El acuario contenía cuatro microhábitats del mismo tamaño $(0,5 \times 0,75 \mathrm{~m})$ para todas las densidades probadas. Los microhábitats de vegetación (Egeria densa, 1 planta / $\left.0.01 \mathrm{~m}^{2}\right)$ y canto rodado $(70-160 \mathrm{~mm})$ constituyeron los microhábitats con refugio, y los microhábitats de grava $(2-38,5 \mathrm{~mm})$ y una superficie de piedra plana que imitó los hábitats de arcilla consolidada que la especie utiliza, constituyeron los microhábitats sin refugio. Para diferenciar las preferencias por microhábitat, se ubicó un microhábitat sin refugio al lado de uno con refugio. Los cuatro microhábitats elegidos para la experimentación se dispusieron de acuerdo a los ambientes donde se encontró frecuentemente esta especie en sistemas fluviales y lacustres (E. Habit observación personal).
DISEÑO EXPERIMENTAL

Para investigar el efecto de la densidad de $G$. plate $i$ en el uso de microhábitat se probó con densidades de 4, 8, 12 y 16 peces, con peces entre 81 y $115 \mathrm{~mm}$ de longitud total. No es posible clasificar estos individuos como juveniles o adultos, ya que se desconoce la talla de primera madurez sexual de la especie. Se midió la disponibilidad de refugio por individuo a una razón de 1:2, 1:4, 1:6 y 1:8. La selección de los peces ya aclimatados fue aleatoria sin remplazo. Las tallas y pesos entre experimentos se mantuvieron iguales $\left(\chi^{2}=2,6, p=0,626 ; \chi^{2}=5,6, p=0,23\right.$ respectivamente), con una talla promedio $98,1 \pm 1,3 \mathrm{~mm}$ y un peso de 7,9 $\pm 3,3 \mathrm{~g}$ (Fig. 1). Para observar la conducta de uso de refugio durante el día, cada experimento tuvo una duración de dos días, con observaciones cada 10 minutos entre 09:00 y 13:00 h y entre 15:00 y 18:00 h, las que no presentaron diferencias significativas entre ambos horarios para las densidades 4, 8 y 16 (ANOVA, $F=2,145, p=0,095 ; F=0,696, p=0,555$ y $\mathrm{F}=2,361, \mathrm{p}=0,072)$. Para la densidad 12 sí hubo diferencias significativas en el uso de hábitat entre ambos horarios $(\mathrm{F}=19,401, \mathrm{p}=0,000)$. Sin embargo, en este experimento lo que se compara es el uso de hábitat entre distintas densidades, por lo que se utilizan los datos de ambos horarios agregados.

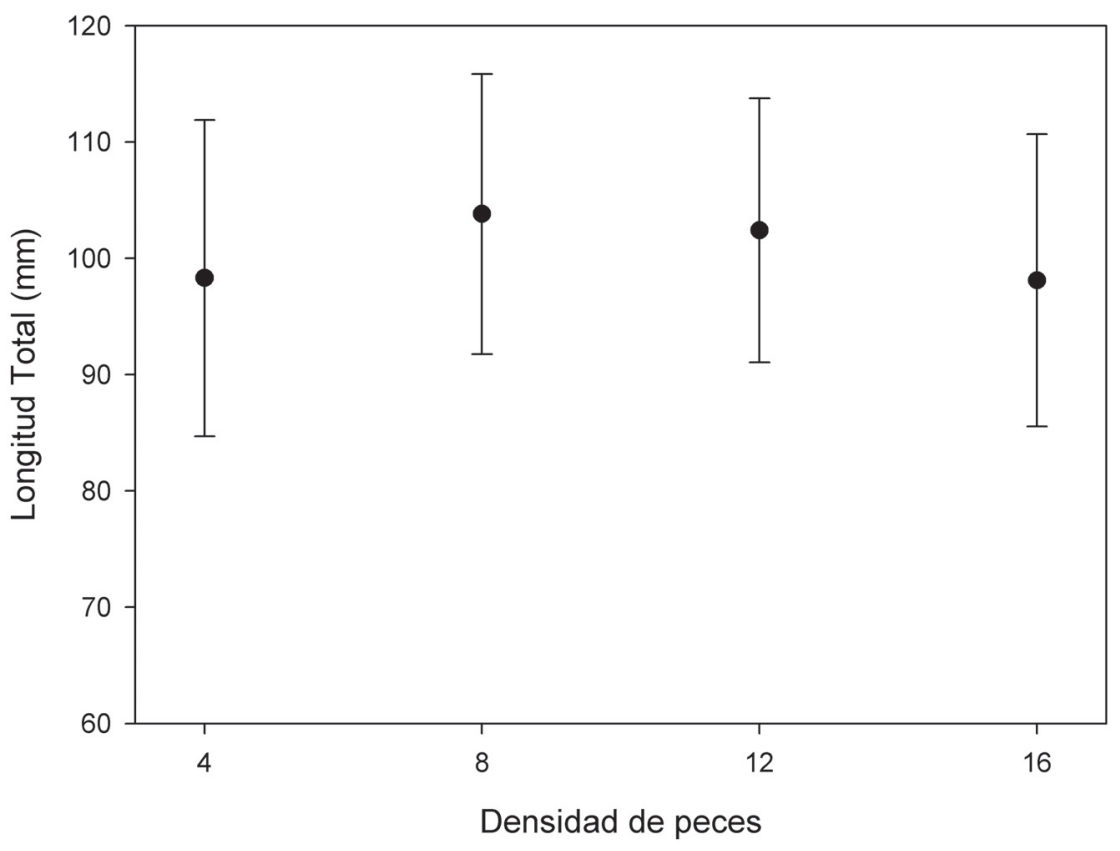

FIgURA 1. Longitud total de Galaxias platei por densidad utilizados en los experimentos (media \pm desviación estándar).

FIGURE 1. Total length of Galaxias platei by density used in the experiments (average \pm standar deviation). 


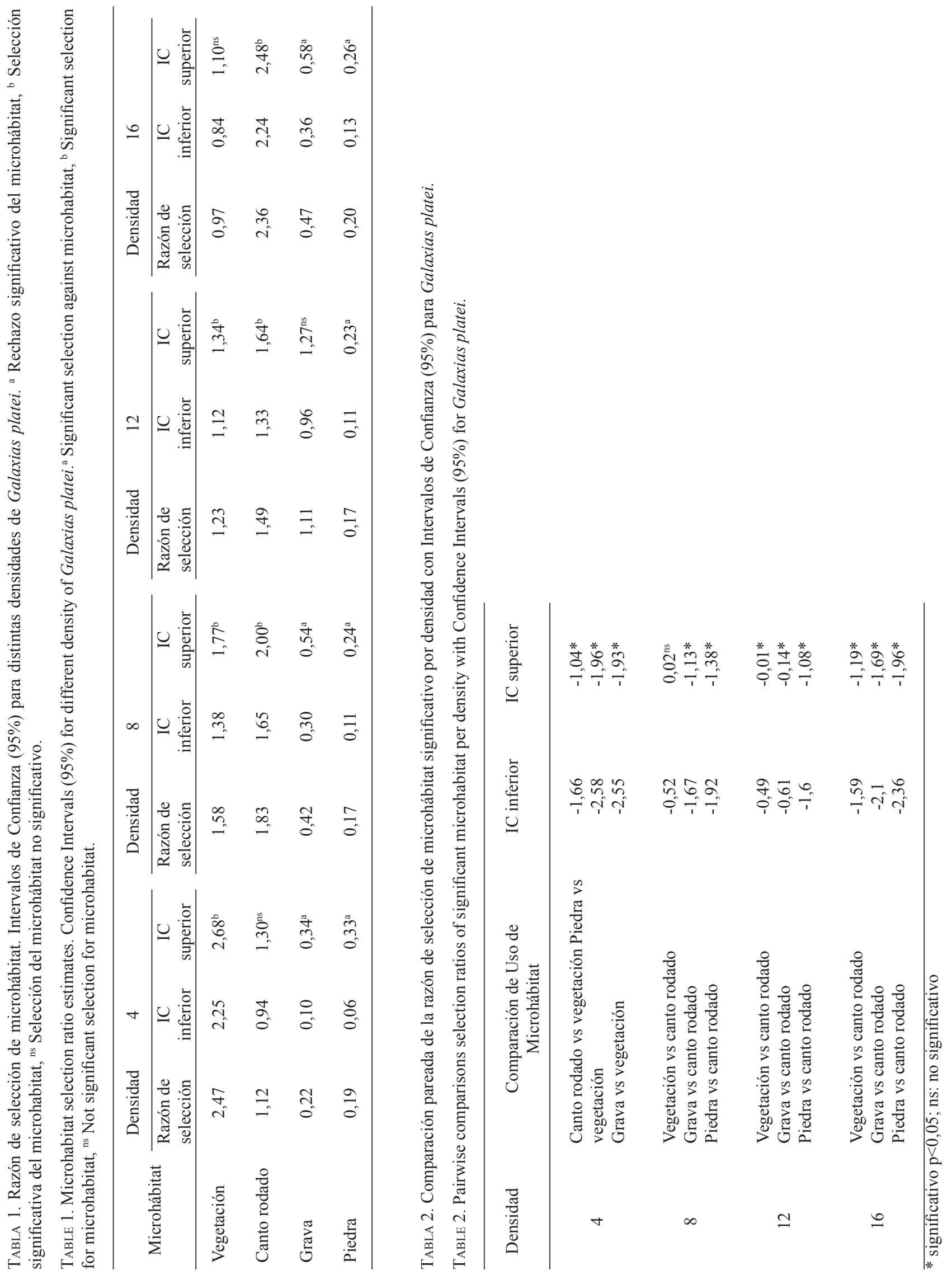


En cada ensayo se contó el número de peces que ocupaba cada microhábitat, mediante observación directa y con apoyo de cámaras para su posterior revisión y validación de las observaciones. Se consideró un protocolo estándar de observación, para evitar efectos del observador sobre el comportamiento de los peces (Vanderpham et al. 2012). Para asegurar una buena aclimatación, en cada ensayo los peces fueron ubicados en el acuario el día anterior al experimento, sin ser alimentados. Se evaluó la razón de selección de microhábitat como la razón entre la proporción de uso y la proporción de microhábitat disponible (Manly et al. 2002; Smith et al. 2011) como:

Razón de selección $=\left(\mathrm{n}_{\mathrm{i}} / \mathrm{N}\right) /\left(\mathrm{v}_{\mathrm{i}} / \mathrm{V}\right)$

Donde:

$\mathrm{n}_{\mathrm{i}}=$ número de peces observados en el parche de hábitat $\mathrm{i}$.

$\mathrm{N}=$ número de peces totales.

$\mathrm{v}_{\mathrm{i}}=$ volumen del parche de hábitat $\mathrm{i}$.

$\mathrm{V}=$ volumen total de hábitat disponible.

Una razón de selección cercana a 1 indica no selectividad para un tipo de microhábitat. Valores altos de razón de selección indican preferencia por un tipo de microhábitat, mientras que valores pequeños indican rechazo al tipo de microhábitat (Manly et al. 2002).

\section{ANÁLISIS ESTADÍSTICO}

En todas las densidades probadas, para determinar si un microhábitat fue seleccionado según su disponibilidad, se aplicó un test $\chi^{2}$. Se realizó un total de 88 observaciones en cada parche por cada tratamiento, resultando un total de 1408 observaciones. Se evaluó si un microhábitat era seleccionado, no seleccionado o rechazado mediante intervalos de confianza de la razón de selección donde se consideró una selección significativa cuando los intervalos no incluyeron el valor 1 (95\%) (Manly et al. 2002). Por último, se realizó una comparación pareada entre la razón de selección entre los microhábitats, mediante intervalos de confianza, verificando la significancia de la diferencia entre las medias observadas.

\section{RESULTADOS}

A todas las densidades ensayadas, la selección de microhábitat por G. platei no dependió de su disponibilidad, la cual fue siempre constante (i.e.: $\chi^{2}=148,8-243,4-62,1$ y 134,$8 ; \mathrm{p}<0,001$, para densidades de $4,8,12$ y 16 peces, respectivamente).

Para la densidad de 4 peces, la mayor frecuencia de uso de microhábitat fue de un $61,7 \%$ en el de vegetación (Fig. 2a). La razón de selección del microhábitat y su intervalo de confianza, y el análisis de comparación pareada muestran que a esta densidad hubo una selección significativa por el microhábitat de vegetación (Tablas 1 y 2), de rechazo por los de grava y de piedra plana, y un uso no significativo en el canto rodado (Tablas 1 y 2). Los peces que utilizaron el parche de canto rodado, se encontraban en la superficie o en los intersticios del sustrato.

Para la densidad de 8 peces, la mayor frecuencia de uso observada fue de un $46 \%$ en el canto rodado, seguido por vegetación con una frecuencia de uso de un 39\% (Fig. 2b). La selección del microhábitat fue significativa en el canto rodado y vegetación (Tablas 1 y 2), y de rechazo por la grava y piedra plana (Tabla 1). El análisis de comparación pareada muestra que no hubo diferencias significativas entre la selección del canto rodado y vegetación (Tabla 2).

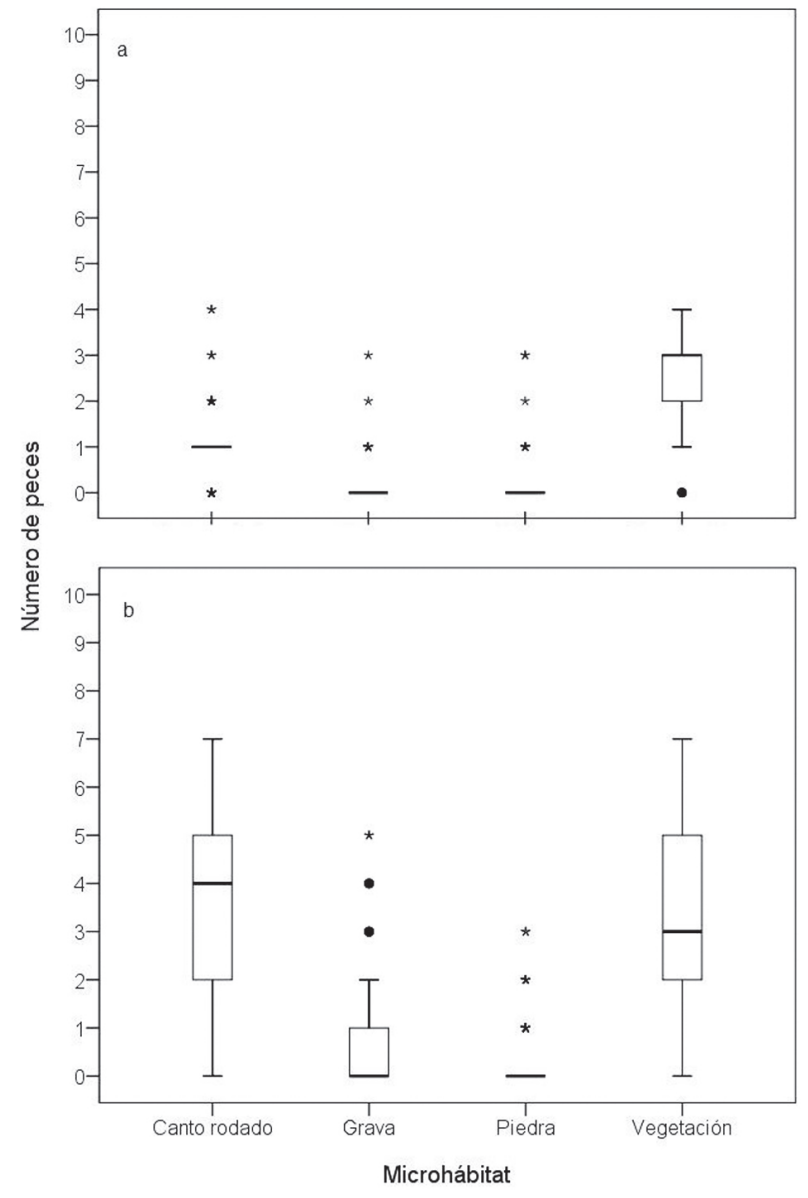

Figura 2. Diagrama de cajas para el uso de cuatro microhábitats por Galaxias platei (letra $\mathrm{a}=4$ peces; letra $\mathrm{b}=8$ peces). La línea gruesa corresponde a la mediana y los símbolos a los valores atípicos $(\bullet=1,5$ a 3 veces el rango intercuartil) y extremos $(\star=$ mayor a 3 veces el rango intercuartil).

FIGURE 2. Side-by-side boxplots of microhabitat use for Galaxias platei (letter $\mathrm{a}=4$ fish; letter $\mathrm{b}=8$ fish). The gross line represents the median and the symbols are outliers $(\bullet=1,5$ and 3 times the interquartile range) and extreme values ( $\star$ more than 3 times the interquartile range). 
Para la densidad de 12 peces, la mayor frecuencia de uso observada fue de un $37 \%$ en el canto rodado seguido por vegetación con una frecuencia de uso de un 30\% (Fig. 3a). La selección del microhábitat se mantuvo significativa en el canto rodado y vegetación (Tablas 1 y 2), y de rechazo por grava y piedra plana (Tabla 1). El análisis de comparación pareada mostró que el microhábitat de canto rodado fue seleccionado con una mayor probabilidad que los otros microhábitats (Tabla 2).

Para una densidad de 16 peces, la mayor frecuencia de uso observada fue de un $59 \%$ en el canto rodado (Fig. 3b). La selección del microhábitat fue significativa en el canto rodado (Tablas 1 y 2), y de rechazo por la grava y de piedra

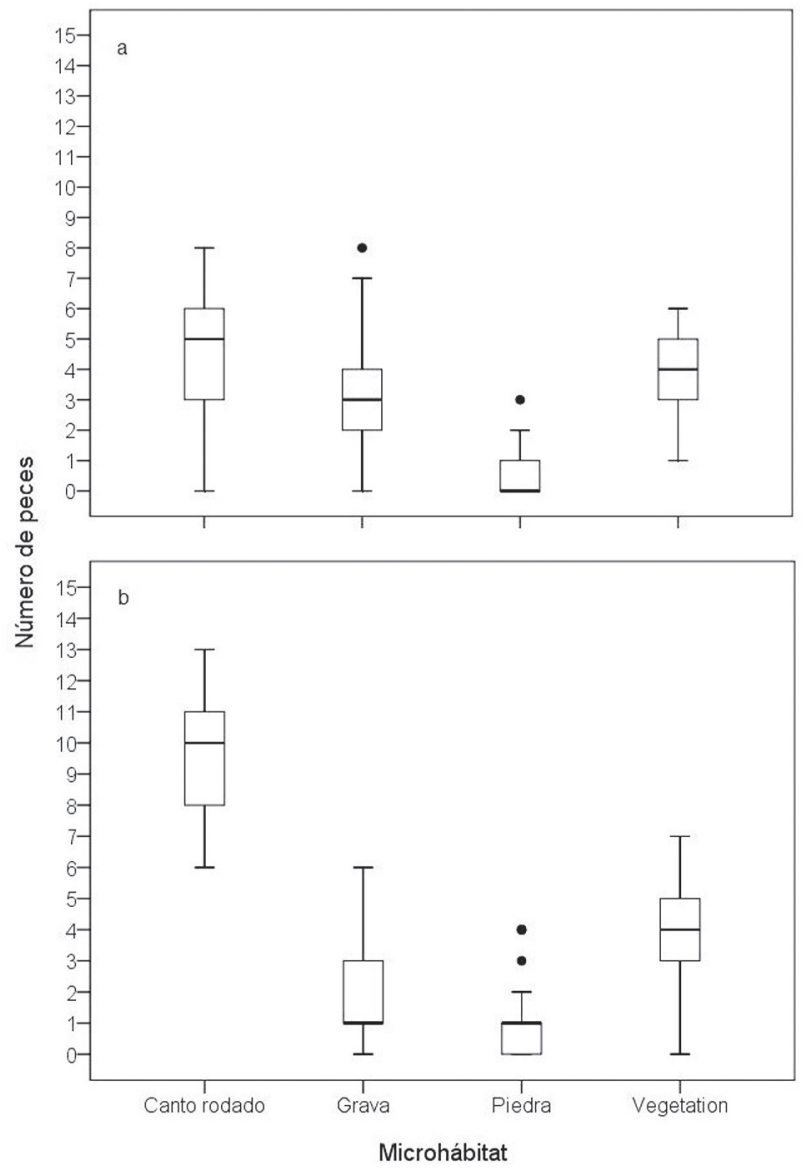

FIgURA 3. Diagrama de cajas para el uso de cuatro microhábitats por Galaxias platei (letra $\mathrm{a}=12$ peces; letra $\mathrm{b}=16$ peces). La línea gruesa corresponde a la mediana y los símbolos a los valores atípicos $(\bullet=1,5$ a 3 veces el rango intercuartil) y extremos $(\star=$ mayor a 3 veces el rango intercuartil).

FIgURE 3. Side-by-side boxplots of microhabitat use for Galaxias platei (letter $\mathrm{a}=12 \mathrm{fish}$; letter $\mathrm{b}=16$ fish). The gross line represents the median and the symbols are outliers $(\bullet=1,5$ and 3 times the interquartile range) and extreme values ( $\star$ more than 3 times the interquartile range). plana, y un uso no significativo de vegetación (Tablas 1 y 2). El análisis de comparación pareada mostró que el microhábitat de canto rodado fue seleccionado con una mayor probabilidad que los otros microhábitats (Tabla 2).

\section{DISCUSIÓN}

En este estudio encontramos que en todas las densidades probadas, G. platei selecciona microhábitats con refugio y que el aumento de la densidad provoca un cambio en la preferencia desde sustratos con vegetación hacia canto rodado. Por el contrario, en todas las densidades probadas, los microhábitats sin refugio, tanto de grava como de piedra plana, fueron rechazados significativamente.

Aunque la selección de hábitat puede ser más compleja en hábitats naturales que lo representado en este estudio experimental, los resultados mostraron que $G$. platei no seleccionó los microhábitat de manera aleatoria. Si bien estos hallazgos se restringen a sólo cuatro tipos de microhábitat, ellos fueron seleccionados sobre la base de los hábitats que esta especie utiliza en la naturaleza (Habit et al. 2012; García et al. 2012a). Igualmente, las densidades seleccionadas para realizar el experimento, se basaron en abundancias conocidas para la especie. En este sentido, $G$. platei presentan una gran variabilidad en sus abundancias dentro de su amplia distribución. Así por ejemplo, la hemos encontrado en densidades tan bajas como un individuo cada $100 \mathrm{~m}^{2}$ (Habit et al. en preparación) hasta densidades mucho mayores en lagos Patagónicos donde es la única especie presente (e.g. Lagos Yulton y Thompson, Habit et al. 2010, 2012). En estos últimos, su densidad es comparable a la mayor ensayada en este estudio $\left(11 \mathrm{ind} / \mathrm{m}^{2}\right)$. Además, este experimento utilizó un rango de tallas de una distribución estadísticamente similar entre las distintas densidades, que en promedio representan individuos de tallas homogéneas que son los característicos habitantes de ambientes someros litorales ( $<1,5 \mathrm{~m}$; Sobenes et al. en preparación).

Nuestros resultados demuestran que el uso de microhábitats con refugio es denso-dependiente, y por ende, es el resultado de interacciones intraespecíficas (Davey et al. 2009; Armstrong \& Griffiths 2001; Rangeley \& Kramer 1998). Tal resultado es esperable para una especie como G. platei, la cual no sólo es altamente piscívora-caníbal (Milano et al. 2006), sino que además es la única especie de pez presente en muchos sistemas lacustres de la Patagonia (Habit et al. 2012). En tal tipo de sistemas monoespecíficos, donde el canibalismo es alto, el uso de hábitats con buenos refugios puede determinar la sobrevivencia de un individuo. Además, el cambio de selección con la densidad, desde microhábitats con vegetación a bajas densidades a aquellos con sustrato grueso (cantos rodados) a densidades mayores, demuestra 
una tendencia al comportamiento gregario, el cual también es un tipo de comportamiento antidepredatorio (Milano et al. 2002). Ello cobra mayor sentido considerando que los experimentos se efectuaron en horario diurno, cuando la principal actividad de la especie es refugiarse (Habit et $a l$., en preparación). A futuro será importante analizar el uso y selección nocturna de hábitat, cuando esta especie se encuentra activa buscando alimento (Habit observación personal).

La conducta que describimos en cuanto al uso de mayor refugio por $G$. platei es similar a la reportada para especies de salmónidos (Young 2004; Amstrong \& Griffiths 2001; Rangeley \& Kramer 1998). En este sentido, es interesante plantearse cómo se manifiesta la relación interespecífica entre dos o más especies (e.g. G. platei y una o más especies de salmónidos) que muestran también evidencia de competencia intraespecífica. Este tipo de estudios experimentales ayudará a entender la interacción interespecífica entre galáxidos nativos y salmonídeos, y por lo tanto, impulsar medidas para asegurar la coexistencia.

Finalmente, otro alcance de este estudio es que sugiere que la disponibilidad de hábitats con refugio es importante para la mantención de poblaciones de G. platei, lo que debe ser considerado en sistemas fluviales y lacustres intervenidos por obras civiles que alteran el hábitat. Por ende, tales proyectos deben incluir medidas de manejo asociadas a la generación y/o restauración de hábitats con refugios apropiados.

\section{AGRADECIMIENTOS}

Los autores agradecen al Consejo Nacional de Investigación, Ciencia y Tecnología CONICYT por el financiamiento otorgado a través de la beca de doctorado nacional CONICYT D-21080436, y de los proyectos CONICYT AT-24110081, FONDECYT 1110441. También, agradecen a la Dirección de Postgrado de la Universidad Católica de la Santísima Concepción, y a la Dirección de Investigación de la Universidad de Concepción, por el financiamiento otorgado a través del proyecto DIUC-Patagonia 210.310.057-ISP.

\section{BIBLIOGRAFÍA}

Armstrong, J. D. \& S. W. Griffiths. 2001. Density-dependent refuge use among over-wintering wild Atlantic salmon juveniles. Jorunal of Fish Biology 58:1524-1530.

Barriga, J. P., M. A. Battini, P. J. Macchi, D. Milano \& V. E. Cussac. 2002. Spatial and temporal distribution of landlocked Galaxias maculatus and Galaxias platei (Pisces: Galaxiidae) in a lake in the South American Andes. New Zealand Journal of Marine and Freshwater Research 36:345-359.
Bonnett, M. L. \& A. R. McIntosh. 2004. The influence of juvenile brown trout (Salmo trutta) on habitat use of inganga (Galaxias maculatus) in a stream simulator. Journal of the Royal Society of New Zealand 34(4):357-367.

Camp, A. L., C. H. Ryer, B. Laurel \& K. Seals. 2011. Effect of nursery habitat on density-dependent habitat selection in juvenile flatfish. Journal of Experimental Marine Biology and Ecology 404:15-20.

Charnov, E. L. 1976. Optimal Foraging: the Marginal Value Theorem. Theoretical Population Biology 9(2):129-136.

Correa, C. \& A. Hendry. 2012. Invasive salmonids and lake order interact in the decline of puye grande Galaxias platei in western Patagonia lakes. Ecological Applications 22(3):828-842.

Crow, S. K., G. P. Closs, J. M. Waters, D. J. Booker \& G. P. WALLIS. 2010. Niche partitioning and the effect of interspecific competition on microhabitat use by two sympatric galaxiid stream fishes. Freshwater Biology 55: 967-982.

Cussac V., S. Ortubay, G. Iglesias, D. Milano, M. Lattuca, J. Barriga, M. Battini \& M. Gross. 2004. The distribution of South American galaxiid fishes: the role of biological traits and post-glacial history. Journal of Biogeography 31(1):103-121.

Cussac V. E., D. A. Fernández, S. E. Gómez \& H. L. López. 2009. Fishes of southern South America: a story driven by temperature. Fish Physiology and Biochemistry 35(1):2942.

Davey, A. J., S. J. Hawkins, G. F. Turner \& C. P. Doncaster. 2005. Size-dependent microhabitat use and intraspecific competition in Cottus gobio. Journal of Fish Biology 67:428-443.

Davey, A. J., C. P. Doncaster \& O. D. Jones. 2009. Distinguishing between interference and exploitation competition for shelter in a mobile fish population. Environmental Modeling and Assesment 14:555-562.

Fretwell, S. \& H. Lucas. 1970. On territorial behavior and other factors influencing habitat distribution in birds. I: theoretical development. Acta Biotheoretica 19:16-36.

García, A., J. GonzÁlez \& E. Habit. 2012a. Caracterización del hábitat de peces nativos en el río San Pedro (cuenca del río Valdivia, Chile). Gayana 76 (Número Especial):36-44.

García, A., C. Sobenes, O. Link \& E. Habit. 2012b. Bioenergetic models of the threatened darter Percilia irwini. Marine and Freshwater Behaviour and Physiology 45(1):17-28.

García de Leaniz C., G. Gajardo \& S. Consuegra. E. 2010. From the Best to Pest: changing perspectives on the impact of exotic salmonids in the southern hemisphere. Systematics and Biodiversity 8(4):447-459.

Griffiths, S. W. \& J. D. Armstrong. 2002. Rearing conditions influence refuge use among over-wintering Atlantic salmon juveniles. Journal of Fish Biology 60:363-369.

Habit, E., P. Piedra, D. E. Ruzzante, S. J. Walde, M. Belk, V. Cussac, J. González \& N. Colin. 2010. Changes in the distribution of native fishes in response to introduced species and other anthropogenic effects. Global Ecology and Biogeography 19(5):697-710.

Habit, E., J. González, D. E. Ruzzante \& S. J. Walde. 2012. Native and introduced fish species richness in Chilean Patagonian lakes: inferences on invasion mechanisms 
using salmonid-free lakes. Diversity and Distributions: 18:1153-165.

Jara-Seguel, P., I. Valdebenito \& C. Palma-Rojas. 2008. Contenido de ADN nuclear en Galaxias platei (TELEOSTEI: OSMERIFORMES: GALAXIIDAE). Gayana 72(2):196-201.

MacArthur, R. H. \& E. R. Pianka. 1966. On optimal use of a patchy environment. The American Naturalist 100 (916):603-609.

Manly, B., L. McDonald, D. Thomas, T. McDonald \& W. ERICKSON. 2002. Resource selection by animals. Kluwer Academic Publishers, Dordrecht. 219 pp.

MCDowaLl, R. M. 2006. Crying wolf, crying foul, or crying shame: alien salmonids and a biodiversity crisis in the southern cool-temperate galaxiid fishes? Reviews in Fish Biology and Fisheries 16:233-422.

McIntosh A. R., T. A. Crowl \& C.R. Townsed. 1994. Size-related impacts of introduced brown trout on the distribution of native common river galaxias. New Zealand Journal of Marine and Freshwater Research 28:135-144.

McIntosh A. R., P.A. McHugh, N. R. Dunn, J. M. Goodman, S. W. Howard, P. G. Jellyman, L. K. O’Brien, P. Nyström \& D. J. WoODFORD. 2010. The impact of trout on galaxiid fishes in New Zealand. New ZealJournal of Ecology 34(1):195206.

Milano, D., V. E. Cussac, P. J. Macchi, D. E. Ruzzante, M. F. Alonso, P. H.Vigliano \& M. A. Denegri. 2002. Predator associated morphology in Galaxias platei in Patagonian lakes. Journal of Fish Biology 61: 138-156.

Milano, D., D. E. Ruzzante, V. E. Cussac, P. J. Macchi, R. A. Ferriz, J. P. Barriga, J. C. Aigo, M. E. Lattuca \& S. J. WALDE. 2006. Latitudinal and ecological correlates of morphological variation in Galaxias platei (Pisces, Galaxiidae) in Patagonia. Biological Journal of the Linnean Society 87:69-82.

Penaluna, B. E., I. Arismendi \& D. Soto. 2009. Evidence of Interactive Segregation between Introduced Trout and Native Fishes in Northern Patagonian Rivers, Chile. Transactions of the American Fisheries Society 138:839845.

Pitcher, T.J. 1983. Heuristic definitions of fish shoaling behaviour. Animal Behaviour 31:611-613.

Rangeley, R. W. \& D. L. Kramer. 1998. Density-dependent antipredator tactics and habitat selection in juvenile pollock. Ecology 79(3):943-952.

Ruzzante, D. E., S. J. Walde, J. C. Gosse, V. E. Cussac, E. Habit, T. S. ZemlaK \& E. D. M. Adams. 2008. Climate control on ancestral population dynamics: insight from patagonian fish phylogeography. Molecular Ecology 17(9):22342244

Smith, D. M., S. A. Welsh, \& P. J. Turk. 2011. Selection and preference of benthic habitat by small and large ammocoetes of the least brook lamprey (Lampetra aepyptera). Environmental Biology of Fish 91:421-428.

Sobenes, C., A, García, E. Habit, \& O. Link. 2012. Mantención de peces nativos dulceacuícolas de Chile en cautiverio: un aporte a su conservación ex situ. Boletín de Biodiversidad de Chile 7:27-41.

Townsend, C. R. 2003. Individual, Population, Community, and Ecosystem Consequences of a Fish Invader in New Zealand Streams. Conservation Biology 17(1):38-47.

Uglem, I., E. Kuørsvik, K. Gruven \& A. Lamberg. 2009. Behavioural variation in cultivated juvenile Atlantic cod (Gadus morhua L.) in relation to stocking density and size disparity. Applied Animal Behaviour Science 117:201209.

Vanderpham, J. P., S. Nakagawa \& G. P. Closs. 2012. Diel variation in use cover and feeding activity of a benthic freshwater fish in response to olfactory cues of a diurnal predatori. Environmental Biology of Fishes 93:547-556.

Werner, E. E., J. F. Gilliam, D. J. Hall \& G. G. Mittelbach. 1983. An experimental test of the effects of predation risk on habitat use in fish. Ecology 64(6):1540-1548.

Young, K. A. 2004. Asymmetric competition, habitat selection, and niche overlap in juvenile salmonids. Ecology 85(1):134149.

Zemlak, T. S., E. M. Habit, S. J. Walde, M. A. Battini, E. D. M. Adams \& D. E. Ruzzante. 2008. Across the southern Andes on fin: glacial refugia, drainage reversals and a secondary contact zone revealed by the phylogeographical signal of Galaxias platei in Patagonia. Molecular Ecology 17(23):5049-5061.

Zemlak, T. E., S. J. Walde, E. Habit \& D. E. Ruzzante. 2011. Climate-induced changes to the ancestral population size of two Patagonian galaxiids: the influence of glacial cycling. Molecular Ecology 20(24):5280-5294.

Recibido: 08.05.12

Aceptado: 05.11.12 